\title{
Application of Dynamic Database in the DPSA of Equipment System
}

\author{
Xin $\operatorname{Ren}^{1,2, a}$ \\ ${ }^{1}$ Graduate School, National Defense University, Beijing, 100091, China \\ ${ }^{2}$ Medical Protection Laboratory, Naval Medical Research Institute, Shanghai, 200433, China \\ aemail: renxins841013@163.com
}

Keywords: DPSA, dynamic database, equipment system, reliability

\begin{abstract}
To compare with the traditional PSA, the DPSA (dynamic PSA) can well solve the problems containing dynamic factors of time, process variables personnel operating behavior. The reliability data is the basis of DPSA, and the quality of DPSA largely depends on the quality of input data. The system composition, working process and analysis calculation model of DPSA will be introduced in this paper, and the application of dynamic database and DPSA interaction techniques in the equipment system also will be discussed for the first time. The DPSA scheme based on the "instructions-reply" has been presented according to the mathematical model of DPSA, which will promote the application and development of DPSA technology in the equipment system.
\end{abstract}

\section{Introduction}

Dynamic probabilistic safety assessment (DPSA) is the systematic method of equipment system safety assessment by means of probability theory. Reliability data and reliability parameters are the foundations of DPSA, and the quality of DPSA depends largely on the quality of input data. The main purpose of establishing the dynamic reliability database of equipment system is to further improve the safety level and device management level of equipment system and provide the basic reliability parameters for the dynamic probability safety assessment and reliability centered maintenance of equipment system.

\section{DPSA system and dynamic database}

DPSA system is the daily safety management system which updates the model in order to reflect the current or planning configuration based on the risk model and information support system and evaluates the system configuration change with using the model. DPSA system is composed of DPSA model and DPSA tools and dynamic database. DPSA system composition and working process can be shown in figure 1 and figure 2 respectively as follows.

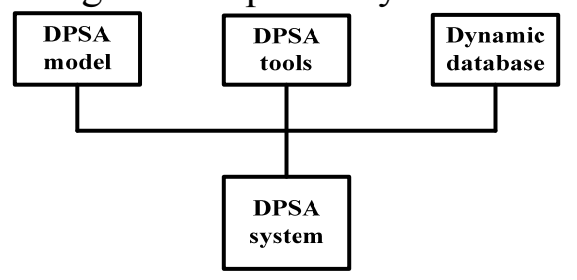

Fig.1 Constitute of DPSA system 


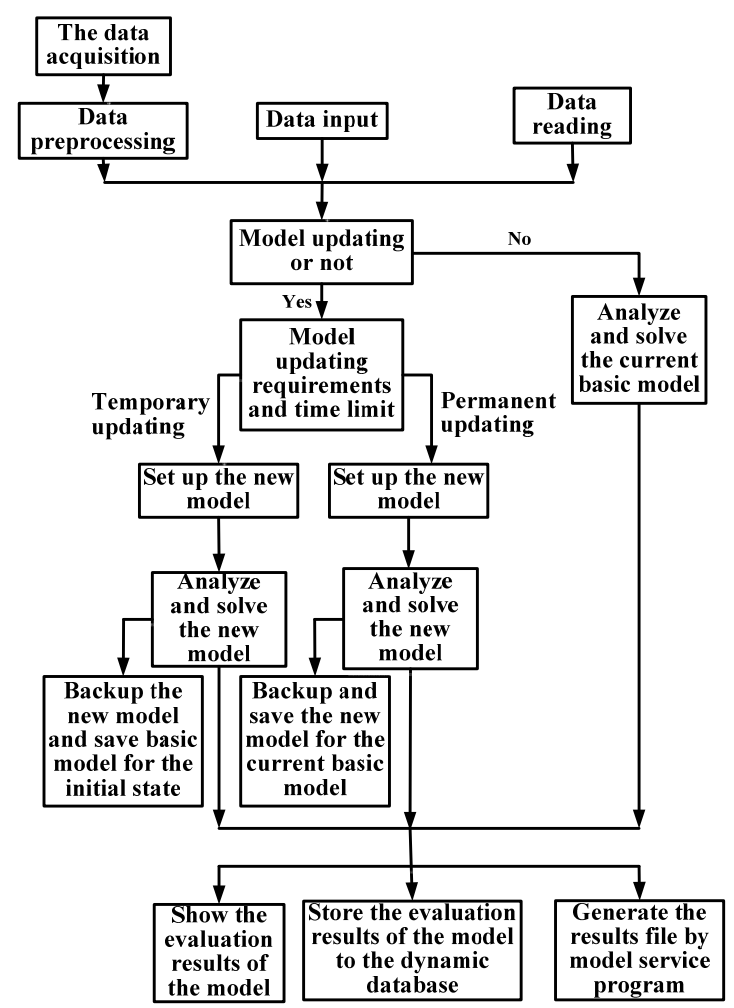

Fig. 2 The working flow chart of DPSA system

Equipment system dynamic database consists of three parts: data acquisition system, data pretreatment system and data storage system.

\section{DPSA analysis model and implementation technology of dynamic database}

\section{(1) DPSA calculation model}

The PSA of any equipment system is a response to its design and operation, however, PSA data that is the basis of the analysis will change over time, and so in order to ensure that PSA can always provide reasonable results, PSA must be converted to DPSA. The conclusion can be obtained by ESD and FTA analysis method that the equipment system is very complex and a variety of initial events can lead to the failure occurrence of equipment component. The failure probability of the equipment component can be calculated by the following formula.

$$
\lambda_{y}(t)=\sum_{x \in E^{0}} \lambda_{x}(t) p_{y / x}(t)
$$

Among them, $\lambda_{y}(t)$ is the failure probability of equipment component at $t$ time, $\lambda_{x}(t)$ is the incidence rate of initial event at $\mathrm{t}$ time, $\mathrm{E}^{0}$ is the initial event set, $p_{y / x}$ is the failure probability of equipment component when the initial event occurs at t time, and the value of $p_{y / x}$ can be obtained by the quantization of ESD/FTA. $p_{y / x}(\mathrm{t})$ can be represented as the following form.

$$
p_{y / x}(t)=f\left(q_{x_{i}}(t)\right), \mathrm{i}=1 \sim \mathrm{n}
$$

$p_{y / x}(\mathrm{t})$ is the incidence rate of event $\mathrm{y}$ when the initial event $\mathrm{x}$ occurs, and it reflects the response of the equipment security system when the initial event occurs. Whether the security system can realize its predetermined function is determined by the state of equipment in the security system. In the operation process of equipment system, security system equipment may not be available due to failure and it will affect the function realization of equipment security system. How to reflect the fault, repair and maintenance events in the calculation of $p_{y / x}(\mathrm{t})$ is the major problem that needs to be solved. The various events of equipment system are divided into explicit events and implicit events in detail. The explicit events are the events that all can be observed,and the implicit events can be divided into time-correlated events and time-uncorrelated events according to the 
relationship with time. The explicit events use observed values directly when the failure probability of equipment is calculated. $q_{x_{i}}(t)$ is the occurrence probability of base event at a given time and it can take 0 or 1 . It will take 1 when the equipment in maintenance is not available and it will take 0 when the equipment is in normal operation For the implicit events, the occurrence probability $q_{x_{i}}(t)$ of time-uncorrelated implicit events can take $q$ of constant value, and the occurrence probability $q_{x_{i}}(t)$ of time-correlated implicit events can be calculated by using " $q+\lambda \mathrm{t}$ " model and the calculation formula can be listed as follows.

$$
q_{x_{i}}(t) \approx q_{x_{i}}(0)+\lambda_{s}(t-T L)+\lambda_{d} \cdot T M
$$

Among them, $q_{x_{i}}(0)$ is the failure probability is not related to time, $\lambda_{s}$ is the hidden failure strength, $\lambda_{d}$ is the demand failure strength, TL is the last test time point, TM is the task time. By using the methods described in literature [4] and bayesian theories, the equipment system internal changes can be considered in two aspects. Firstly, the influence of system equipment system daily operation events on the risk of equipment can be reflected using the method of dividing events. Moreover, the failure rate changes due to the running time growth of equipment system component can cause changes of the importantance degree on equipment system risk contribution, and the changes can be reflected by using the bayesian theory to deal with reliability parameters. For explicit events, the observations can be used directly, and for implicit events, the computation formula is as follows.

$$
q(t)=\int q_{x_{i}}(t) \pi\left(\lambda / H_{t^{-}}\right) d \lambda
$$

Among them, $\pi\left(\lambda / H_{t}\right)$ is the posterior distribution considering the equipment system component

history $H_{t^{-}}$which contains component operation number, failure number, the successful test number and so on. $\pi\left(\lambda / H_{t^{-}}\right)$can be calculated by using bayesian formula as follows.

$$
\pi\left(\lambda / H_{t_{-}}\right)=\frac{\pi(\lambda) L\left(\lambda / H_{t_{-}}\right)}{\int \pi(\lambda) L\left(\lambda / H_{t_{-}}\right) d \lambda}
$$

Among them, $\pi(\lambda)$ is the prior distributionof $\lambda, L\left(\lambda / H_{t_{-}}\right)$is the likelihood function, and the calculation formula of $L\left(\lambda / H_{t_{-}}\right)$is as follows.

$$
L\left(\lambda / H_{t^{-}}\right)= \begin{cases}\exp \left\{-\lambda \sum_{i=1}^{N_{t^{-}}} L_{i}^{1}\right\} & N_{t^{-}}^{\prime}=0 \\ \exp \left\{-\lambda \sum_{i=1}^{N_{t^{-}}} L_{i}^{1}\right\} \times \prod_{i=1}^{N_{t^{-}}^{\prime}}\left(1-\exp \left\{-\lambda L_{i}^{-1}\right\}\right) & N_{t^{-}}^{\nu^{-1}}>0\end{cases}
$$

It is consumed that the equipment system components obey index distribution, $L_{i}^{1}$ and $L_{i}^{-1}$ are respectively the life sequence of component success test and failure test, $N_{t^{-}}^{\prime}$ and $N_{t^{-}}$are respectively the number of component success test and failure test. The prior distribution $\pi(\lambda)$ is the gamma function, and its distribution density is as follows.

$$
f(x, \alpha, \beta)= \begin{cases}\frac{\beta^{\alpha}}{\Gamma(\alpha)} x^{\alpha-1} e^{-\beta x} & x>0 \\ 0 & x \leq 0\end{cases}
$$

Among them, $\Gamma(\alpha)=\int_{0}^{+\infty} x^{\alpha-1} e^{-x} d x, \pi\left(\lambda / H_{t^{-}}\right)$can be calculated by substituting equation (6) and equation (7) into equation (5), and then by substituting equation (3) and equation (4), $p_{y / x}(\mathrm{t})$ can be work out. $\lambda_{x}(t)$ is the occurrence rate of initial events and it can be updated using the bayesian theory, and the calculation formula is similar to equation (5), equation (6) and equation (7), it depends. 
(2) DPSA project based on"instructions - response"

The calculation of $p_{y / x}(t)$ determined by $L_{i}^{1}, L_{i}^{-1}, N_{t^{-}}$and $N_{t^{-}}^{\prime}$ based on DPSA calculation model above, $p(t)$ is determined by the status of security system equipment at t time, $L_{i}^{1} 、 L_{i}^{-1} 、 N_{t^{-}}$、 $N_{t^{-}}^{\prime}$ is decided by the equipment history before $t$ time. The device status at $t$ time and history state of complete record in real time must be obtained so as to achieve DPSA. A set of DPSA scheme based on "instructions-reply" method can be proposed combined with the DPSA mathematical model, and the scheme canbetter achieve the real-time risk analysis function of equipment system. The essence of "instruction-reply" method is to build an interactive dynamic device database, and the DPSA processing core only can accept "instruction" and "answer" information as shown in figure 3. The database can automaticly record all kinds of events of equipment system through direct communication with operator, and data processing module can update DPSA through real-time query of equipment state and operation history. Interaction diagram of Dynamic database and DPSA system can be shown in figure 4.

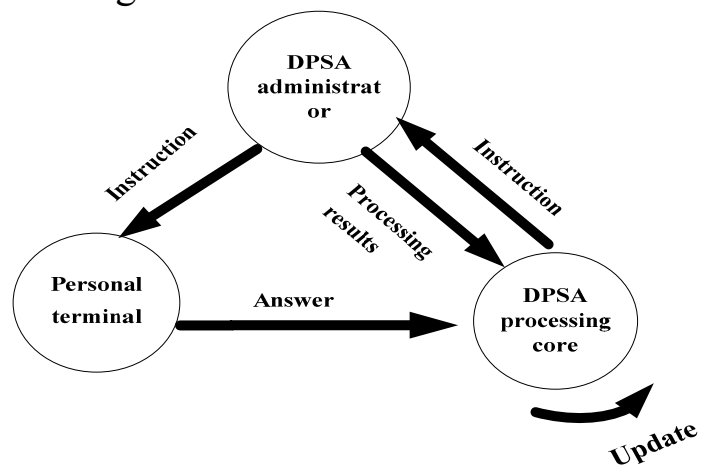

Fig.3 Sketch map of "instructions-reply"method

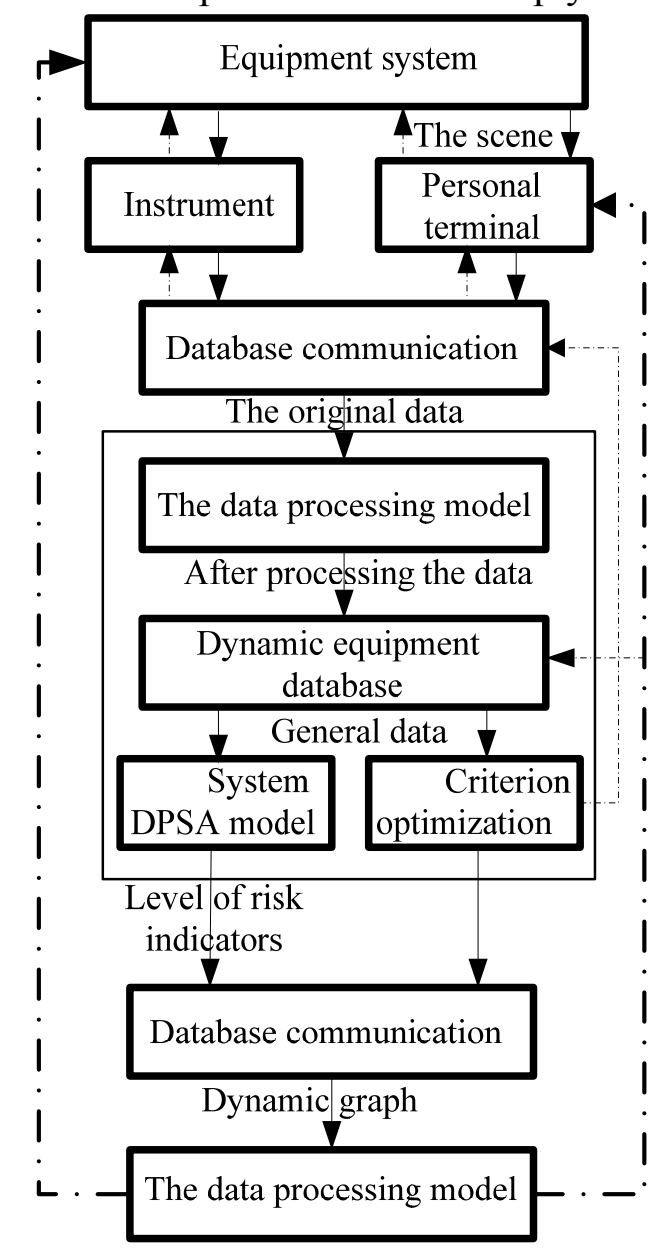

Fig.4 Interaction frame of dynamic data base and DPSA system 


\section{Conclusion}

DPSA is the technology that can constantly update PSA model and record the document so as to correctly reflect the current state of the equipment system. The interaction plan of dynamic data base and DPSA system based on the "instructions-reply" method is put forward according to the mathematical model of DPSA, and the plan can timely reflect the change of the equipment system and realize the real-time risk analysis of the equipment system.

\section{References}

[1] NUCLEAR ENERGY,COMMITTEE ON THE SAFETY OF NUCLEAR INSTALLATIONS.Living PSA development and application in member countries.pp.8-10,1996.

[2]Gunnar Johanson,Jan Holmberg,Safety evaluation by living probability safety assessment:procedures and applications for planning of operational activities and analysis of operating experience.SKI Report94:2,Swedish Nuclear Power Inspectorate,Stockholm,1994.

[3]Living PSA and its use in the Unclear Safety Decision-Making Process,CNSI Technical OpinionPapers, OECD2005,NEANo.4411.

[4]GONZALES D. Network-centric operations case study air-to-air combat with and without Link16[R].Santa Monica Calif: Rand National defense and Research Institute,2005.

[5]TacticaI Digital Infomation Link-TechnicaI Adviceand Lexicon for Enabling Simulation[S].SISO-STD-002-V2.5 DRAFT,2003. 